Supporting Information

\title{
Oxime cross-linked alginate hydrogels with tunable stress relaxation
}

Héctor Sánchez-Morán ${ }^{1}$, Armin Ahmadi ${ }^{1}$,Bernhard Vogler ${ }^{2}$, Kyung-Ho Roh ${ }^{1, *}$

1: Department of Chemical \& Materials Engineering, University of Alabama in Huntsville, Huntsville, AL, 35899, USA.

2: Department of Chemistry, University of Alabama in Huntsville, Huntsville, AL, 35899, USA. 
a)

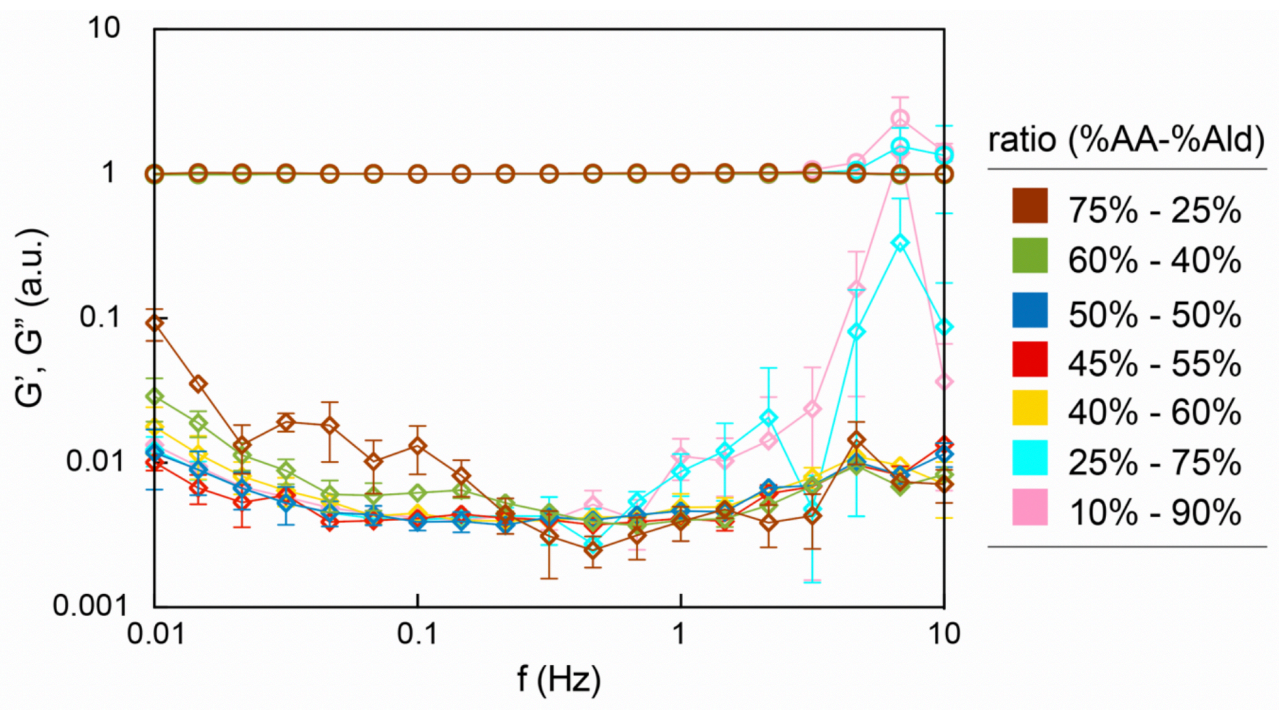

b)

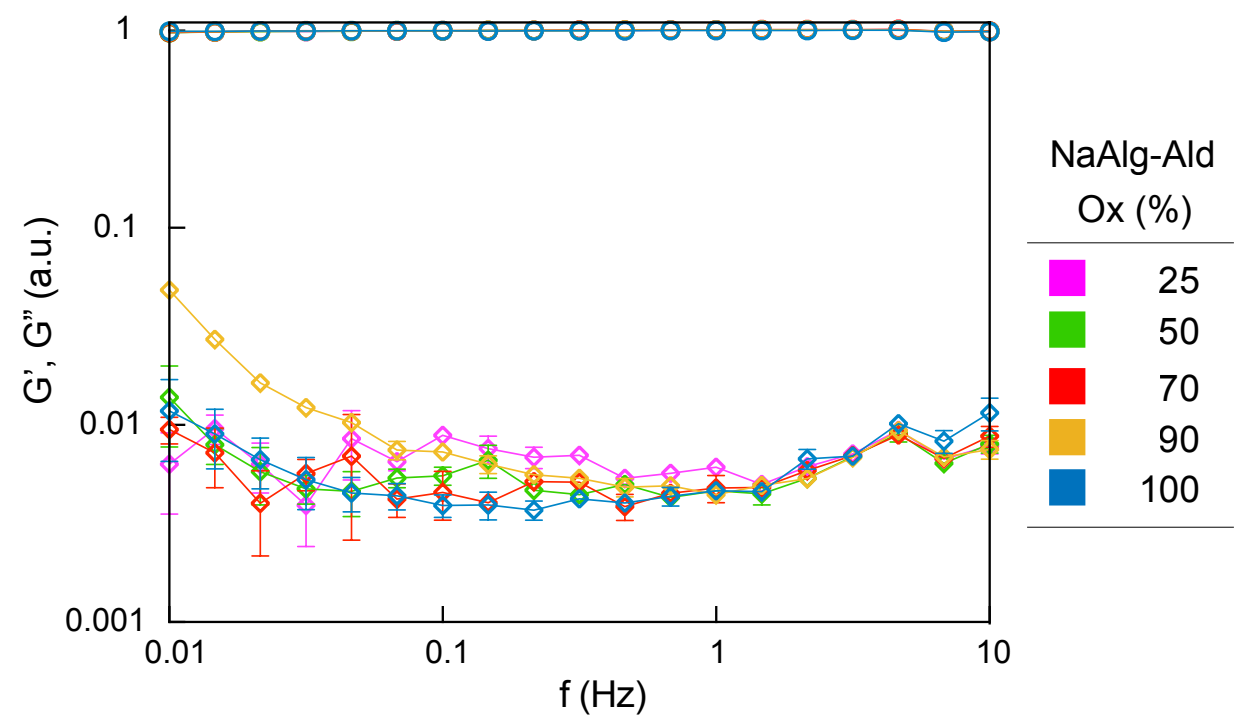

Figure S1. a) Frequency sweeps of hydrogels at 5\% (w/v) concentration for different AA-to-Ald ratio. b) Frequency sweeps of hydrogels at $5 \%(\mathrm{w} / \mathrm{v})$ concentration for fixed 1:1 AA-to-Ald ratio and varying DOx of NaAlg-Ald-X. Tests were performed at a fixed stress of $10 \mathrm{~Pa}$. ( $\mathrm{O}$ ) symbol represents G', and $(\diamond)$ symbol represents G'. All the values were normalized to the G' of each hydrogel at $\mathrm{f}=0.1 \mathrm{~Hz}$. The data represent the mean and the error bars represent the standard error of the mean $(\mathrm{SEM})$ of three different gels $(n=3)$ for each data point. 


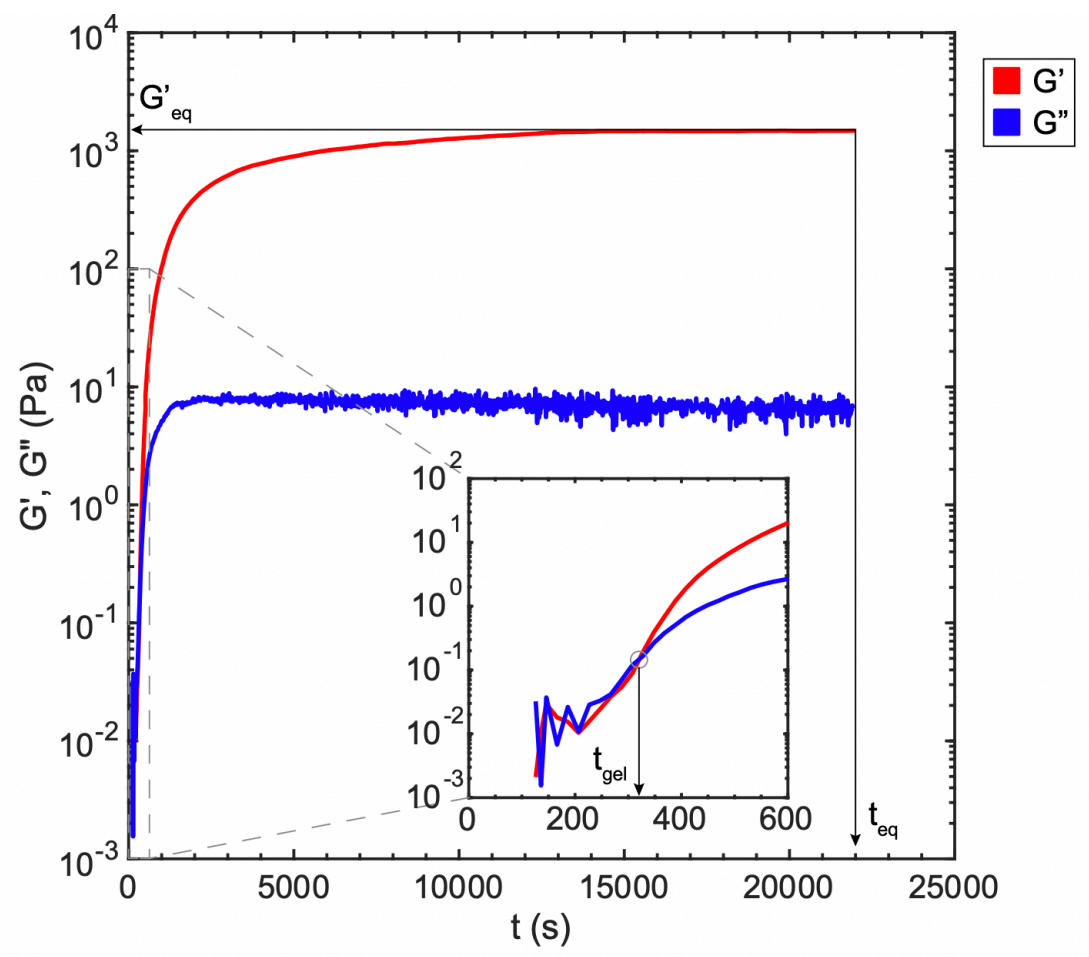

Figure S2. An example of gelation profile and the determination of $\mathrm{t}_{\mathrm{gel}}, \mathrm{t}_{\mathrm{eq}}$ and $\mathrm{G}_{\mathrm{eq}}$.

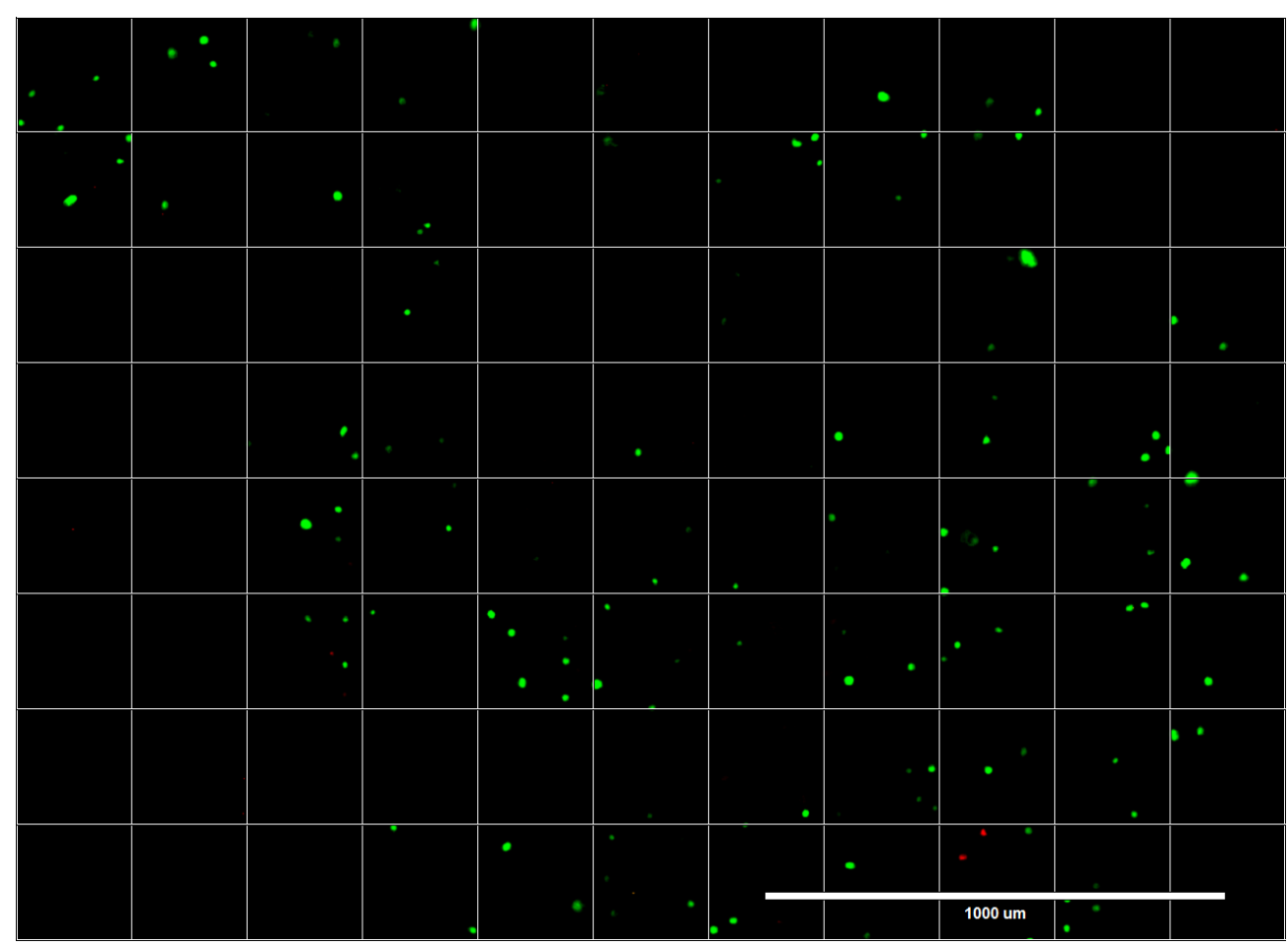

Figure S3. An example fluorescence image with a square grid $\left(250\right.$ x $\left.250 \mu \mathrm{m}^{2}\right)$ for live/dead cell counting. 


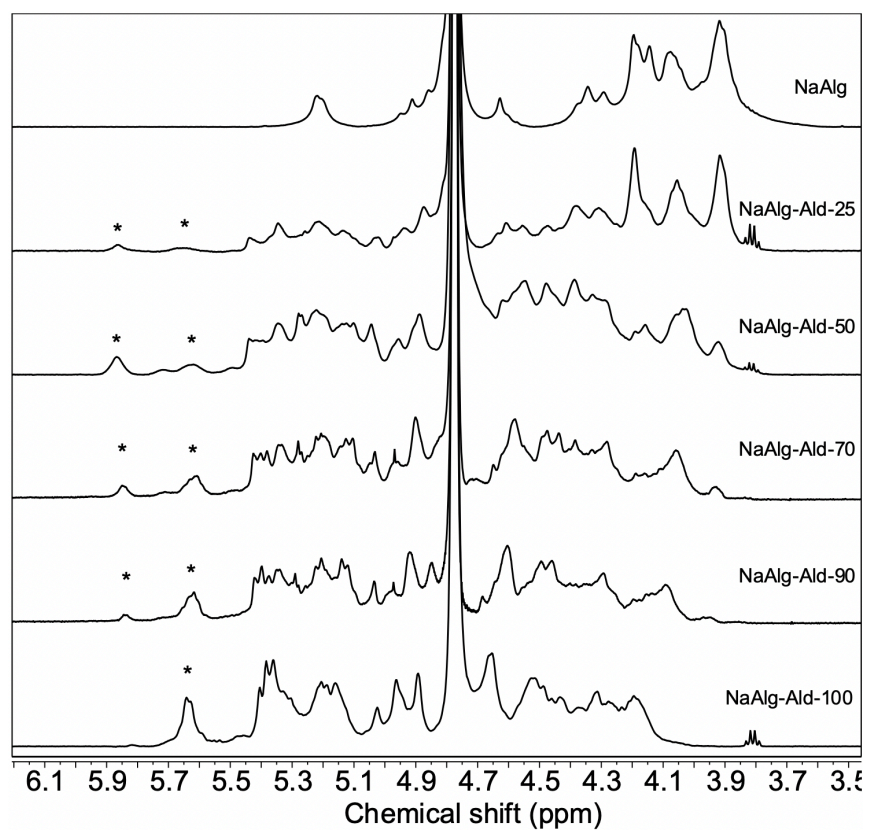

Figure S4. Stacked spectra of NaAlg-Ald-X for $X=25,50,70,90$ and 100\% oxidation. (*) denotes the signals arising from the acetal protons $(\delta=5.65 \mathrm{ppm})$ or hemiacetal protons $(\delta=$ $5.83 \mathrm{ppm})$.

Table S1. Targeted vs. achieved degree of oxidation (DOx) in NaAlg-Ald-X (X is DOx in \%)

NaAlg-Ald degree of oxidation (\%)

\begin{tabular}{lccccc}
\hline Targeted & $25 \%$ & $50 \%$ & $70 \%$ & $90 \%$ & $100 \%$ \\
Achieved & $23.65 \%$ & $50.42 \%$ & $68.47 \%$ & $87.93 \%$ & $100 \%$ \\
\hline
\end{tabular}




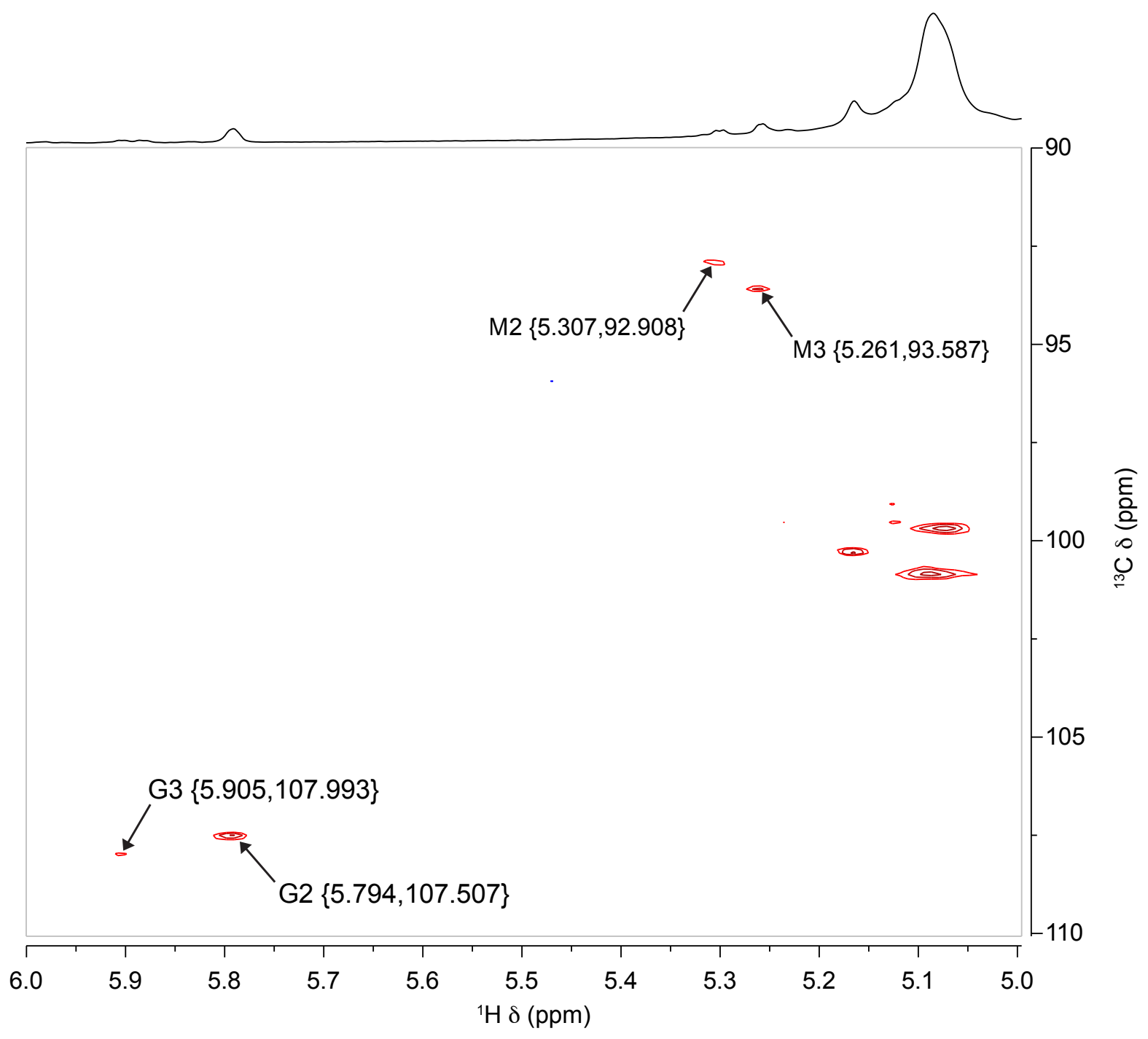

Figure S5. HSQC spectrum of NaAlg-AA. Marked signals represent ${ }^{1} \mathrm{H}_{-}{ }^{13} \mathrm{C}$ correlations at the positions $\mathrm{C}-2$ and $\mathrm{C}-3$ to which alkoxyamines are attached in guluronate and mannuronate conformations. The brackets represent $\left\{{ }^{1} \mathrm{H}\right.$ chemical shift, ${ }^{13} \mathrm{C}$ chemical shift $\}$. 


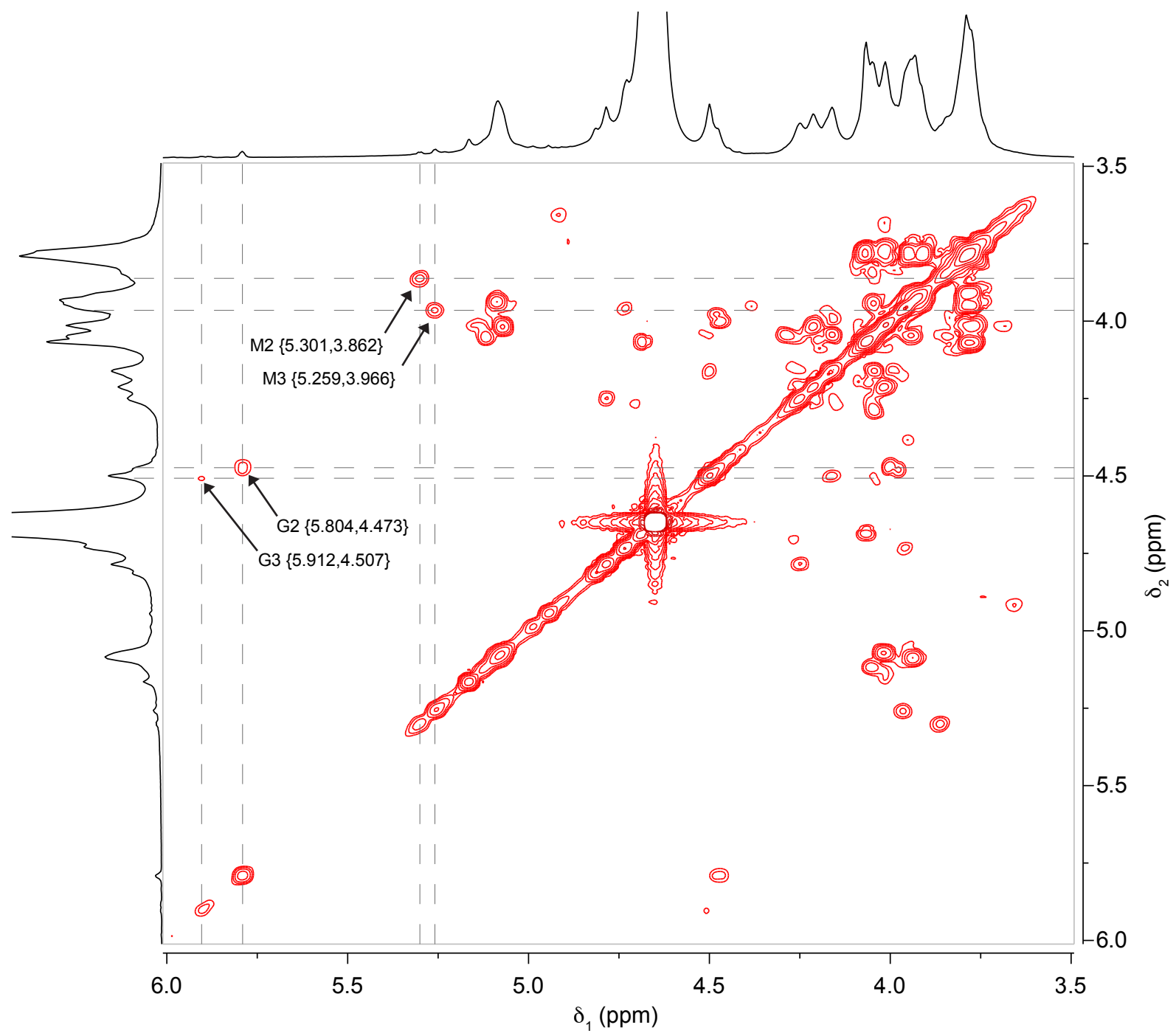

Figure S6. COSY spectrum of NaAlg-AA. Marked signals represent ${ }^{1} \mathrm{H}-{ }^{1} \mathrm{H}$ correlations between protons attached to the carbons $\mathrm{C}-2$ and $\mathrm{C}-3$ to which alkoxyamines are attached in guluronate and mannuronate conformations, and neighboring protons in the same alginate ring spin system. Cross peaks in the second proton channel $\left(\delta_{2}\right)$ underlay below the backbone proton peaks which makes them indistinguishable due to their low intensity. The brackets represent $\left\{{ }^{1} \mathrm{H}\right.$ channel 1 chemical shift, ${ }^{1} \mathrm{H}$ channel 2 chemical shift $\}$. 
a)

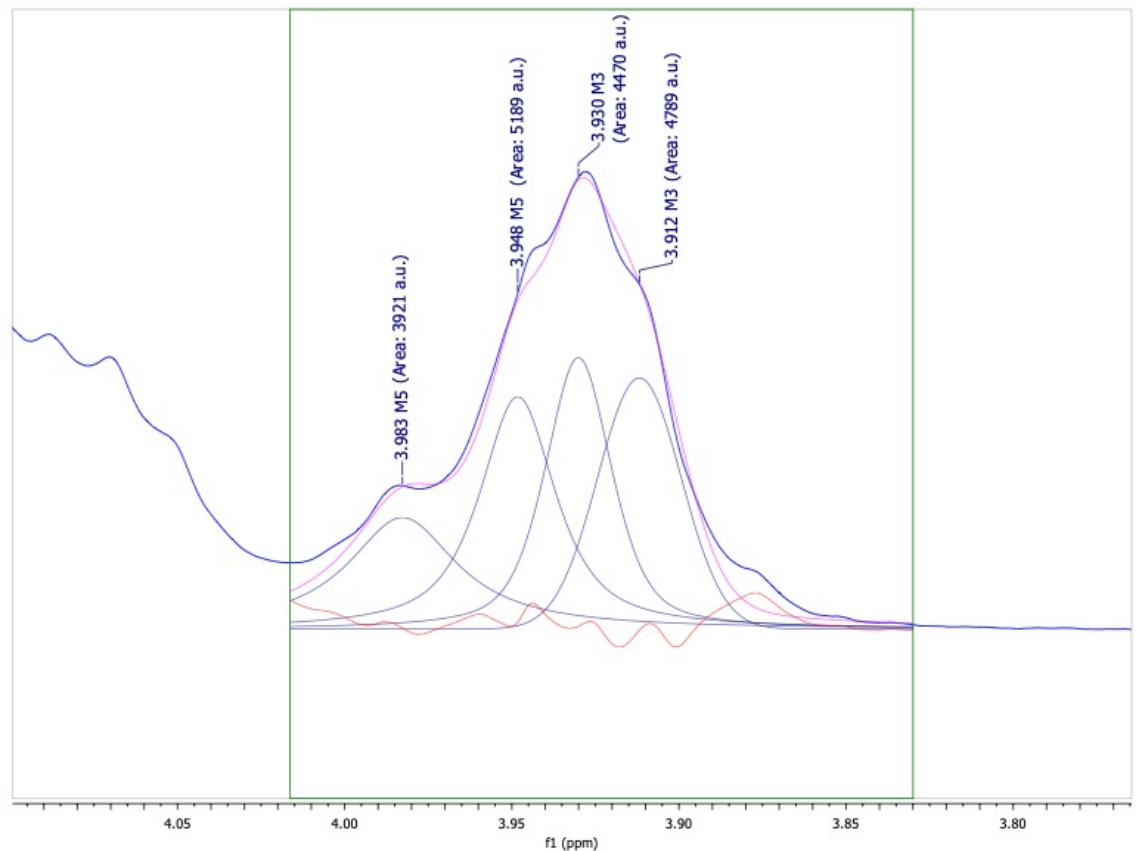

b)

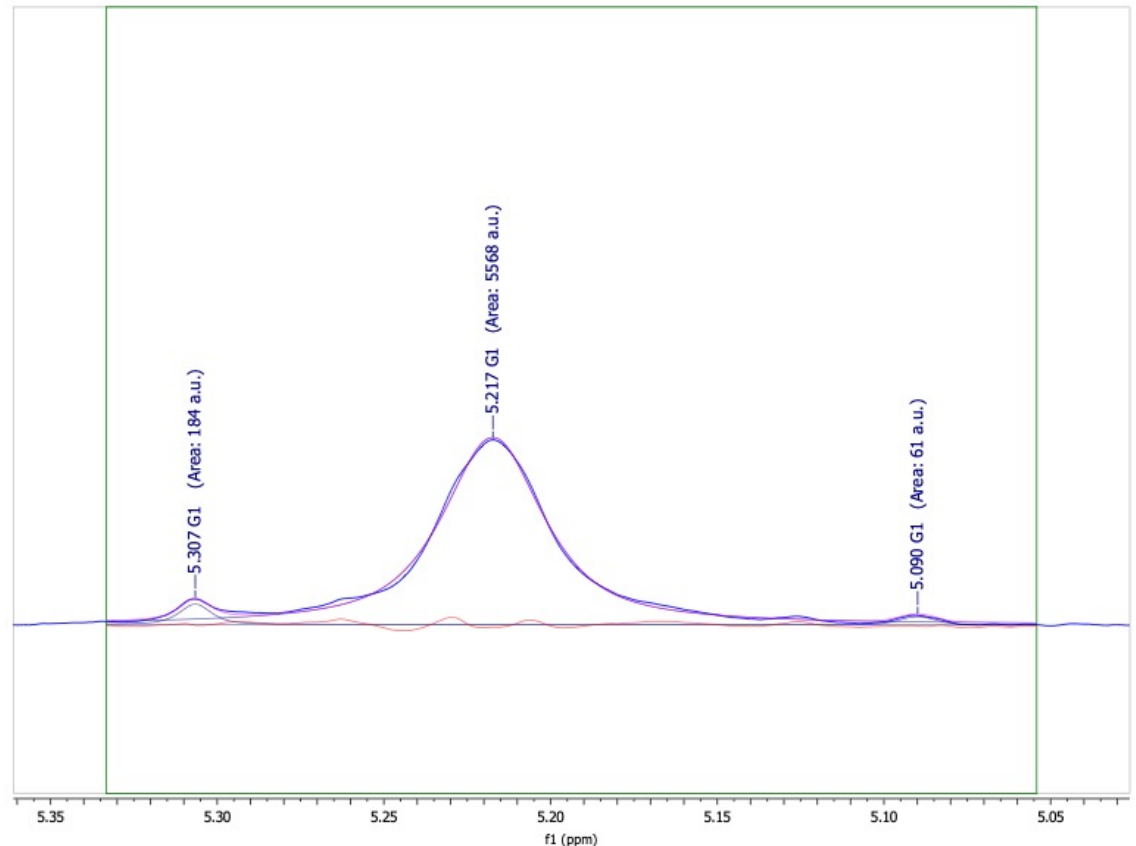


c)

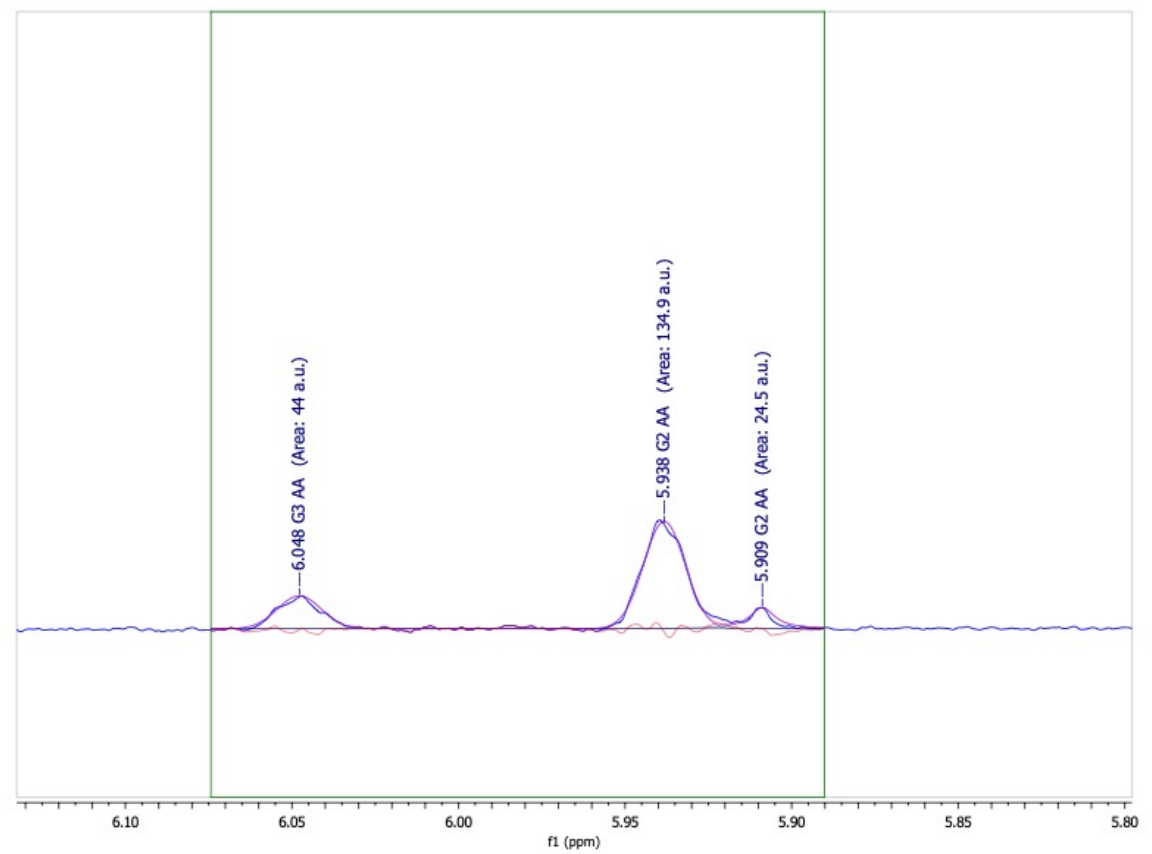

d)

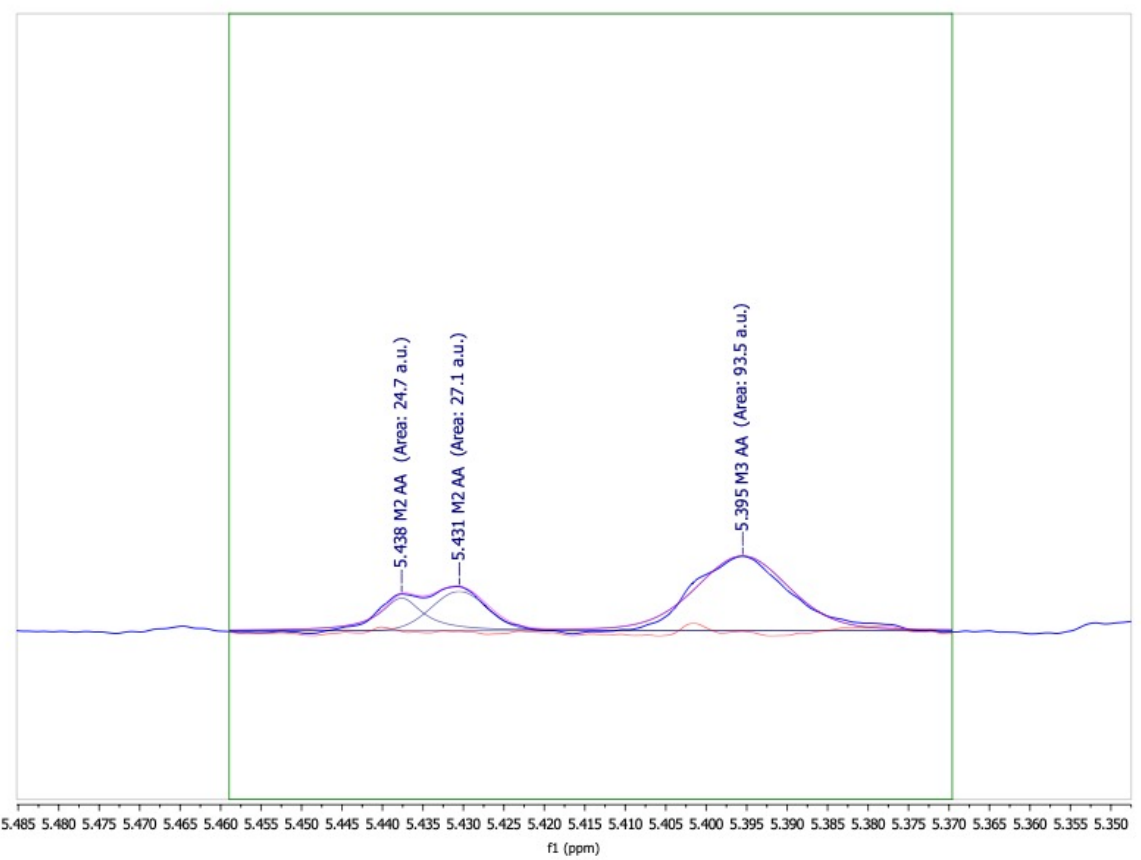

Figure S7. Deconvoluted and fitted ${ }^{1} \mathrm{H}-\mathrm{NMR}$ peaks of NaAlg-AA: a) M3 and M5 protons; b) G1 proton; c) G2-AA and G3-AA protons; d) M2-AA and M3-AA protons. Area under the curves is shown in arbitrary units. 
Table S2. Areas of deconvoluted and fitted peaks in ${ }^{1} \mathrm{H}-\mathrm{NMR}$ spectrum of NaAlg-AA for the calculation of $\mathrm{M} / \mathrm{G}$ ratio and the yield (hydroxyl to alkoxyamine).

\begin{tabular}{ccccccc}
\hline Signal & M3 & G1 & G2-AA & G3-AA & M2-AA & M3-AA \\
Area (a.u.) & 9259 & 5813 & 159.4 & 44 & 51.8 & 93.5 \\
\hline
\end{tabular}

- $\quad \mathrm{M} / \mathrm{G}$ ratio:

$$
\frac{M}{G}=\frac{(\text { Area of } M 3)}{(\text { Area of } G 1)}=\frac{9259}{5813}=1.592
$$

- Hydroxyl to alkoxyamine conversion:

$$
\begin{gathered}
D S=\frac{1}{2} \cdot \frac{\sum(\text { Alkoxyamine peak areas })}{\sum(\text { Polymer backbone areas })} \cdot 100 \\
=\frac{1}{2} \cdot \frac{\left(G 2_{A A}+G 3_{A A}+M 2_{A A}+M 2_{A A}\right)}{(M 3+G 1)} \cdot 100 \\
=\frac{1}{2} \cdot \frac{348.7}{15072} \cdot 100=1.157 \%
\end{gathered}
$$
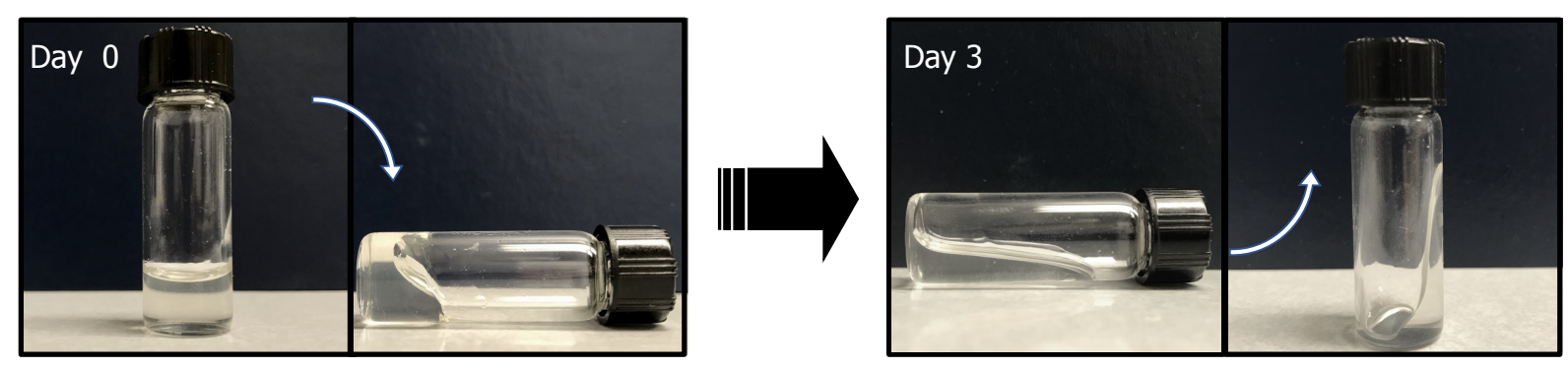

Figure S8. Example of creep behavior displayed by an oxime cross-linked NaAlg hydrogel. The fully formed hydrogel was kept horizontal for three days and changed its shape due to network reorganization. 
a)

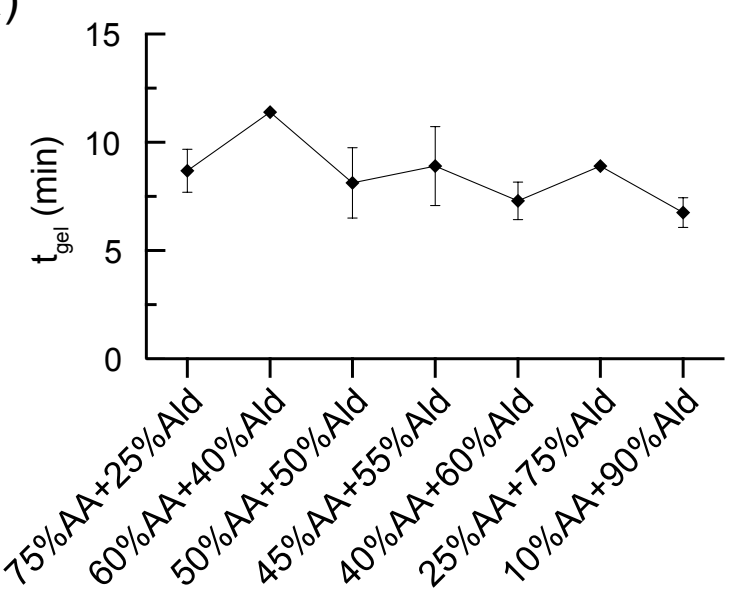

b)

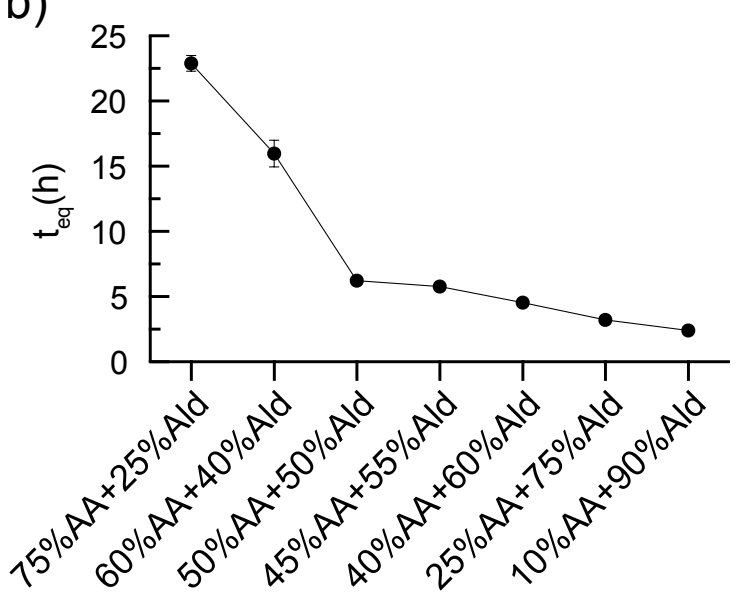

Figure S9. a) $t_{\text {eq }}$ of different hydrogel formulations with varying AA-to-Ald ratio; b) tgel of different hydrogel formulations with varying AA-to-Ald ratio. The data represent the mean and the error bars represent the standard error of the mean (SEM) of three different gels $(n=3)$ for each data point.

Table S3. Fitting parameters of the stretched exponential decay function for hydrogels with different AA-to-Ald ratio. The data represent the mean and the intervals represent the standard error of the mean $(\mathrm{SEM})$ of three different gels $(\mathrm{n}=3)$ for each data point.

\begin{tabular}{crc}
$\begin{array}{c}\text { ratio } \\
\text { (\%AA - \%Ald) }\end{array}$ & $\tau_{k}(\mathrm{~s})$ & $\beta$ \\
\hline $75-25$ & $19.8 \pm 0.3$ & $0.644 \pm 0.002$ \\
$60-40$ & $13.8 \pm 2.7$ & $0.593 \pm 0.020$ \\
$50-50$ & $8.5 \pm 2.2$ & $0.610 \pm 0.005$ \\
$45-55$ & $7.3 \pm 0.6$ & $0.571 \pm 0.004$ \\
$40-60$ & $5.9 \pm 0.7$ & $0.544 \pm 0.007$ \\
$25-75$ & $3.2 \pm 0.6$ & $0.529 \pm 0.021$ \\
$10-90$ & $2.4 \pm 0.2$ & $0.534 \pm 0.008$ \\
\hline
\end{tabular}




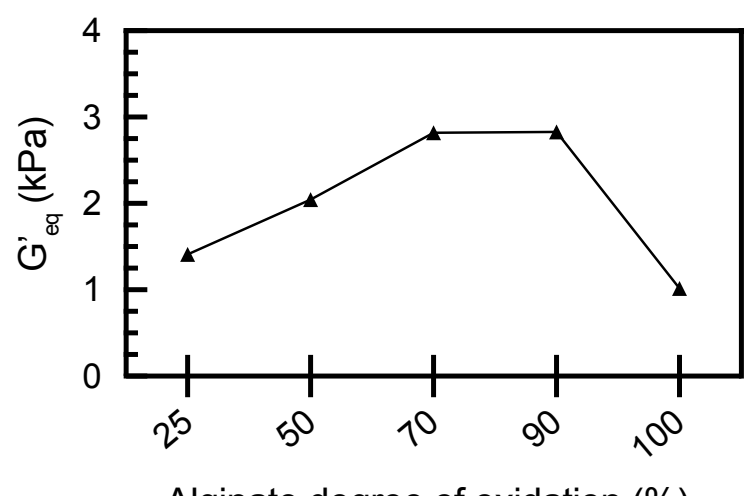

Alginate degree of oxidation (\%)

Figure S10. Storage moduli of the hydrogels made of 3:1 AA-to-Ald mixing ratio at 5\% (w/v) concentration for varying degrees of oxidation (DOx) in NaAlg-Ald-X.

a)

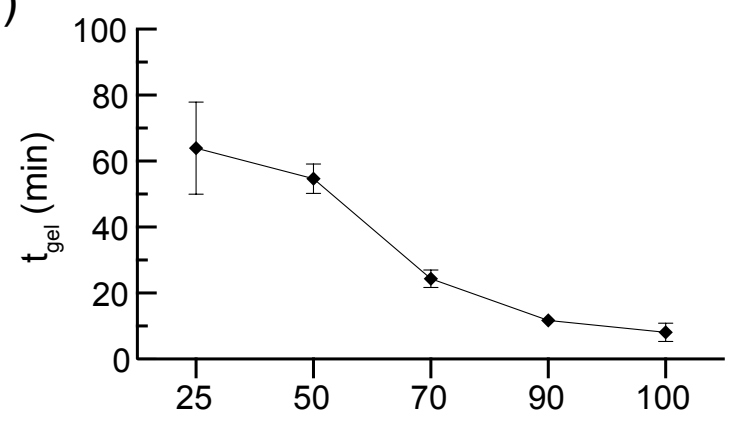

Alginate degree of oxidation (\%) b)

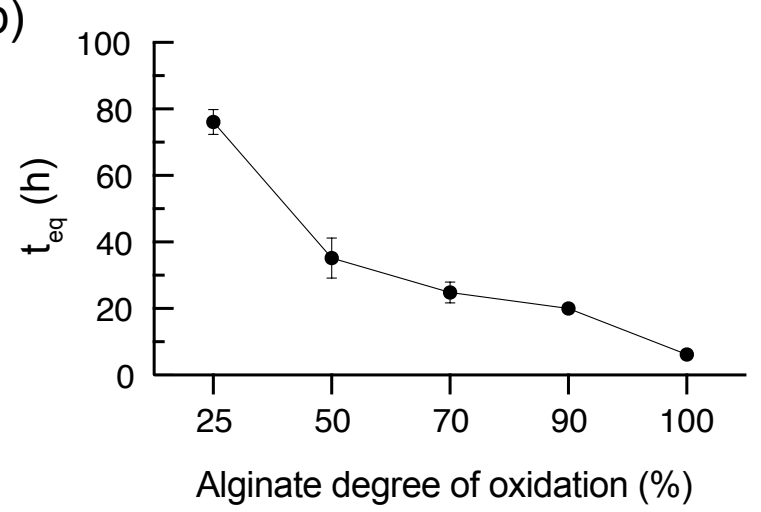

Figure S11. a) $t_{\text {eq }}$ of different hydrogel formulations with varying DOx of NaAlg-Ald-X; b) $t_{\text {gel }}$ of different hydrogel formulations with varying DOx of NaAlg-Ald-X. The data represent the mean and the error bars represent the standard error of the mean $(\mathrm{SEM})$ of three different gels $(n=3)$ for each data point. 
Table S4. Fitting parameters of the stretched exponential decay function for hydrogels with different DOx of NaAlg-Ald-X. The data represent the mean and the intervals represent the standard error of the mean $(\mathrm{SEM})$ of three different gels $(n=3)$ for each data point.

\begin{tabular}{crc} 
DOx $(\%)$ & $\tau_{\mathrm{k}}(\mathrm{s})$ & $\beta$ \\
\hline 25 & $46.8 \pm 5.8$ & $0.743 \pm 0.005$ \\
50 & $40.7 \pm 4.7$ & $0.741 \pm 0.004$ \\
70 & $32.5 \pm 2.8$ & $0.744 \pm 0.016$ \\
90 & $22.2 \pm 2.6$ & $0.711 \pm 0.016$ \\
100 & $8.5 \pm 2.2$ & $0.610 \pm 0.005$ \\
\hline
\end{tabular}

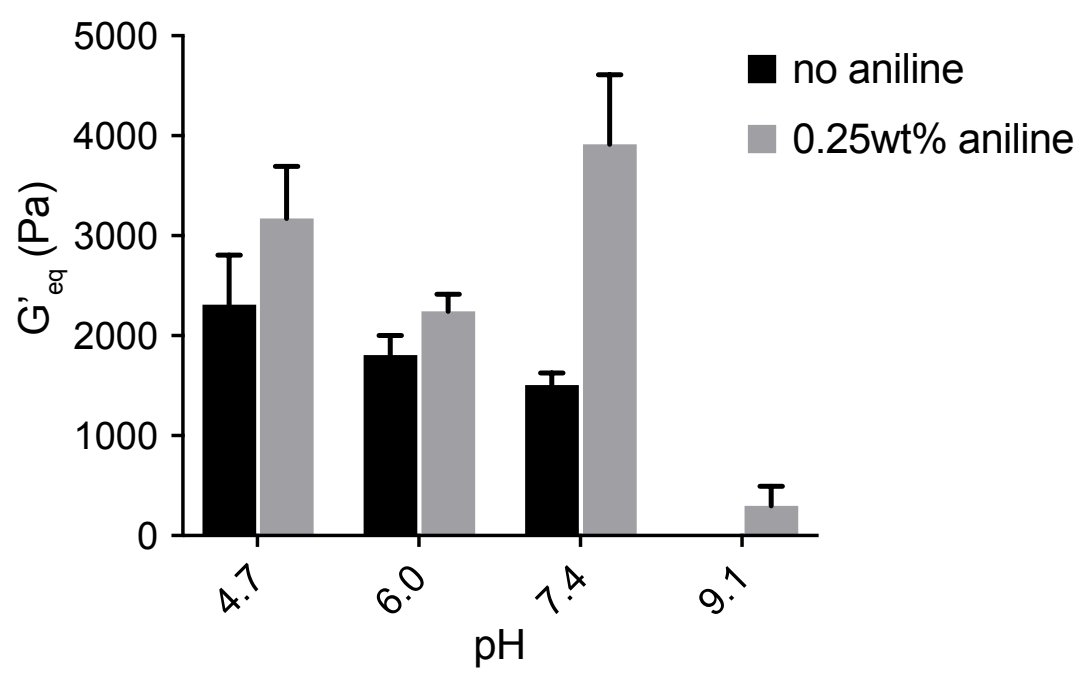

Figure S12. Storage moduli of hydrogels at varying $\mathrm{pH}$ with or without aniline as a catalyst. Bars of the histogram represent the mean and error bars represent the standard error of the mean (SEM) of three different gels for three $(n=3)$ data points. 


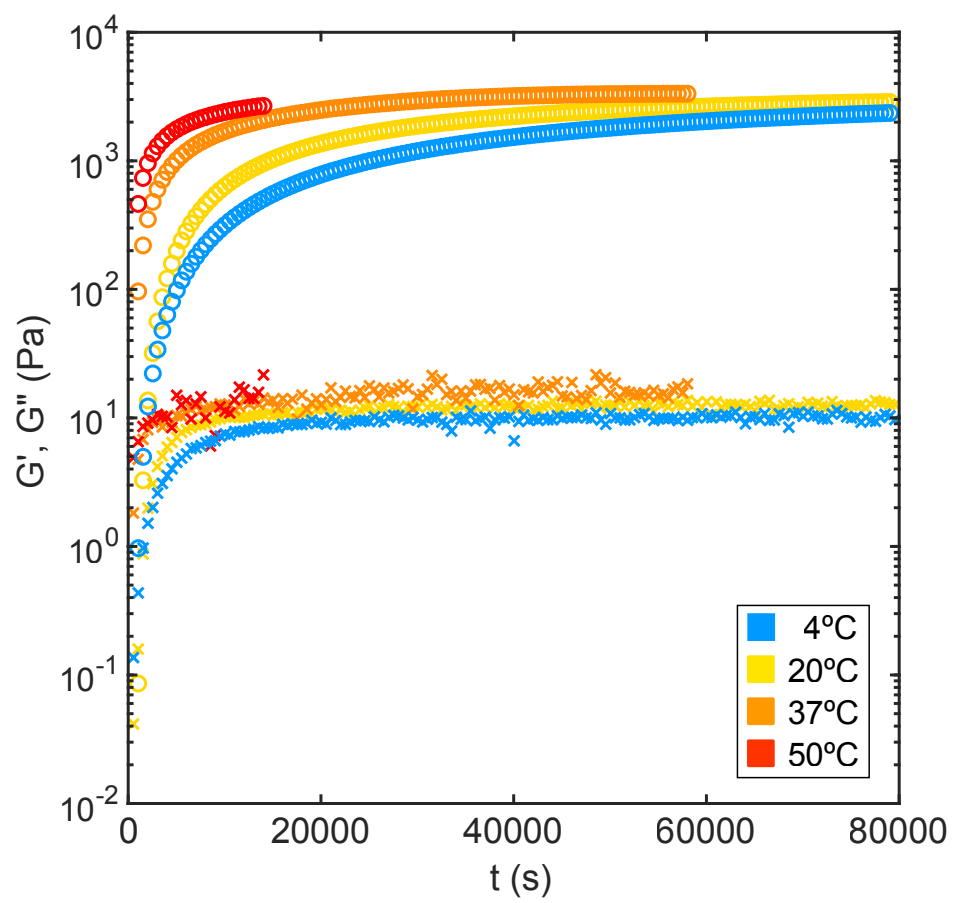

Figure S13. Gelation profiles of hydrogels at different temperatures.
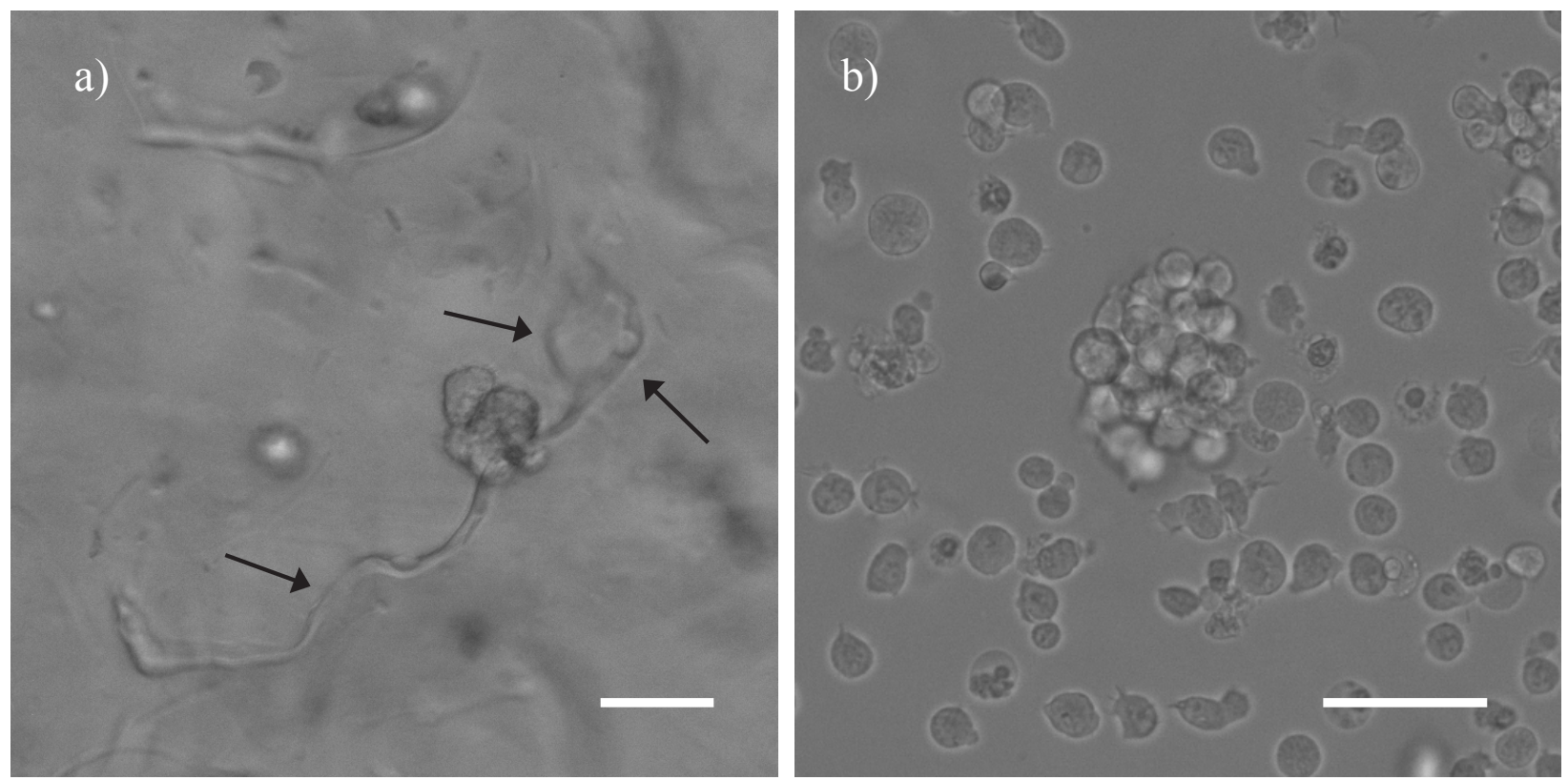

Figure S14. a) An optical microscope image of 2PK-3 B lymphoma cells growing inside of the hydrogel made of 1:1 mixture of NaAlg-AA and NaAlg-Ald-100 at $2 \%(\mathrm{w} / \mathrm{v})$. The cells left migration tracks (arrows) and formed cell clumps. b) An optical microscope image of 2PK-3 cells growing in solution. Scale bars represent $50 \mu \mathrm{m}$. 

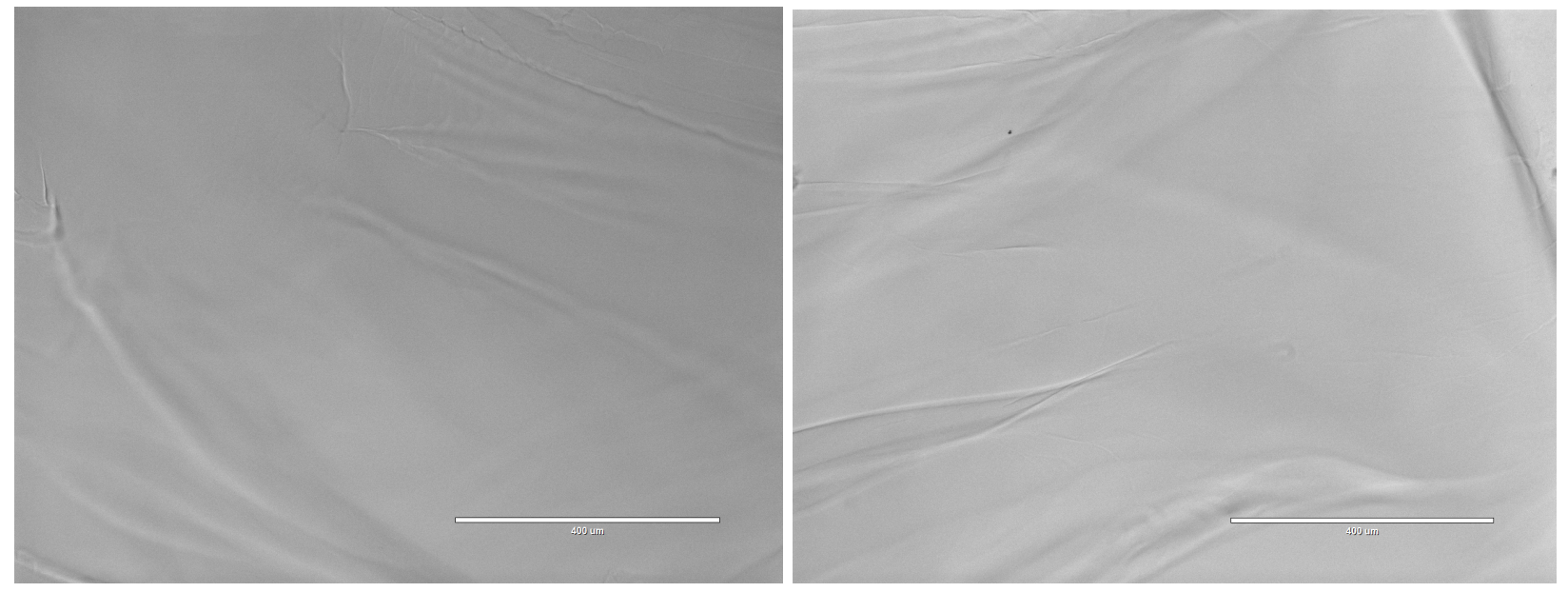

Figure S15. Optical microscope images of hydrogels made of NaAlg-AA and NaAlg-Ald-100 at $2 \%(\mathrm{w} / \mathrm{v})$ without seeded cells, serving as control for verifying that the migration tracks of Figure $5 \mathrm{~b}$ arise from the migrative action of the encapsulated cells. Scale bar represents $400 \mu \mathrm{m}$. 